\title{
Recommendation-Based Trust Model in P2P Network Environment
}

\author{
Yueju Lei and Guangxi Chen \\ Guangxi Key Laboratory of Trusted Software Guilin University of Electronic Technology, \\ Guilin 541004, China \\ chgx@guet.edu.cn, leiyueju_422@sina.com
}

\begin{abstract}
Two kinds of peers' relationship are usually considered for reputation management in P2P network. One of them is direct trust relationship that the reputation is got with two peers interacting directly; the other is recommendation trust that the reputation is got by recommendation of the third party. In this paper, we presented an improved trust model based on recommended in $\mathrm{P} 2 \mathrm{P}$ environment. In order to weight the transaction reputation and recommendation reputation, we introduced a risk value which resists the influence of false recommendation and collaborative cheating from malicious peers. The global reputation value of target peer can be calculated by using the different portions of transaction reputation, recommendation reputation and risk value. At last, we made the simulation experiments verifying the ability of resisting threats such as slandered by malicious peers, collaborative cheating, and so on.
\end{abstract}

Keywords: peer-to-peer network; transaction reputation; recommendation reputation; recommend.

\section{Introduction}

The trust relationship is a core part of interpersonal network relationships. The trust relationship often depends on the direct experience of entity itself to trustees or recommendation experience of other individuals. This relationship with mutual exchanges and mutual trust has formed a trusted network.

In the open P2P network, the relationship of all entities (peers) is coordinate. The open, dynamic and heterogeneous characteristics of P2P network make the entity can enter or leave freely. So, the relationship of peers in network is always dynamic changing. If there is no recommendation of trust third party or trust authority (such as the authentication center (CA)) to participate, it may be difficult to build trust relationships between entities. Therefore, it is very necessary to establish a trust mechanism to measure the credibility of peers in the $\mathrm{P} 2 \mathrm{P}$ network.

\section{Related Works}

Researchers established numerous trust models, which cover the subjective trust and recommend trust [1-2]. W. T. Luke Teacy [3] et al established TRAVOS (Trust and 
Reputation model for agent based on Virtual Organizations) trust model. In this model, trust value is calculated by probability theory, but lack personal experience between agents. Kamvar [4] et al presented a distrusted and secure method to compute global reputation values which is based on Power iteration. By having peers use these global reputation values to choose the peers from whom they download, the network effectively identifies malicious peers from the network. Guo Cheng [5] et al presented a trust evaluation model for quantifying the trustworthiness of peers based on recommendations. They introduced a probability method to relieve the influence of multipath propagation of trust and improved the stability of the result. Li Xiong [6] et al proposed the Peer Trust model(it is abbreviate to PTM) - a reputation-based trust supporting framework, which included a coherent adaptive trust model for quantifying and comparing the trust worthiness of peers based on a transaction-based feedback system over P2P network.

At present, trust model still has some problems and risks in $\mathrm{P} 2 \mathrm{P}$ networks. In response to the above issues, a trust model based on recommended in the network environment is presented in this paper.

\section{Trust Model}

\subsection{Notations}

Here and after, we use the following notations in this paper:

- $\quad \mathrm{i} \rightarrow \mathrm{j}$ The attitude of node $\mathrm{i}$ to node $\mathrm{j}$;

- $\quad \mathrm{i} \leftrightarrow \mathrm{j}$ The transaction of node $\mathrm{i}$ to node $\mathrm{j}$; $D_{i j} \quad$ Direct trust value of $\mathrm{i} \rightarrow \mathrm{j}$

- $\mathrm{R}_{\mathrm{j}}$ Recommendation trust value of node $\mathrm{j}$; $A(x)$ The Accuracy factor

- $R_{x \rightarrow j}$ Feedback information of any node $x$ to node $j$

\subsection{Eigen Trust Model}

The calculation method of trust values of node $\mathrm{j}$ for local trust value in Eigen Trust model [7] is shown as follows:

1) Node i produce a local trust value for $\mathrm{j}$ its direct deals with node $\mathrm{j}$. Let

$$
D_{i j}=S_{i j} / N_{i j}
$$

Where $S_{i j}, F_{i j}$ show the successful transaction times and failure times for node $\mathrm{i} ; \mathrm{N}_{\mathrm{ij}}$ means the total transaction number of $\mathrm{i} \leftrightarrow \mathrm{j}$ in fixed time $\left[\mathrm{t}_{\text {start }}, \mathrm{t}_{\text {end }}\right]$; Sometimes, node i may have different opinion for a transaction. When i think the transaction is successful, but $j$ may think it's not. So here, what we take is the attitude value of trustee to transaction. Obviously, if $\mathrm{N}_{\mathrm{ij}}=0$, then $\mathrm{D}_{\mathrm{ij}}=0$.

2) Set the $R_{x \rightarrow j}$ as the $R_{j}$ feedback to other peers. Let

If $\sum_{\mathrm{x}} \mathrm{S}_{\mathrm{xj}}=0$ orS $\mathrm{xj}_{\mathrm{j}}-\mathrm{F}_{\mathrm{xj}}<0$, thenR $\mathrm{R}_{\mathrm{x} \rightarrow \mathrm{j}}=0$.

$$
=\left(S_{x j}-F_{x j}\right) / \sum_{x} S_{x j}
$$

3) After iterating, we use their global reputation value as the peer's recommended trust in EigenRep model [7]. 


$$
\mathrm{T}_{\mathrm{i} \rightarrow \mathrm{j}}=\sum_{\mathrm{x}} \mathrm{R}_{\mathrm{x} \rightarrow \mathrm{j}} \mathrm{T}_{\mathrm{x}}
$$

In this model, there are two main problems. (1) The model ignores the influence of risk factor; thus $T_{i \rightarrow j}$ that we got may deviate from actual situation. (2) The model uses $T_{i \rightarrow j}$ iterative calculation as $R_{j}$; it doesn't consider the harm of malicious peers.

\subsection{The Recommendation Trust Model}

\section{Direct trust Value}

Definition 1. The local trust value is calculated by transaction experience directly. Take $\mathrm{i} \rightarrow \mathrm{j}$ for an example. The direct trust value is:

$$
=\frac{\alpha S_{i j}}{N_{i j}}+\beta P(x)
$$

Where $\alpha, \beta$ are all the weighted factor; $0 \leq \alpha, \beta \leq 1$, their value depend on the importance of this transaction. $\mathrm{P}(\mathrm{x})$ is punishment factor.

$$
\mathrm{P}(\mathrm{x})=\left\{\begin{array}{rc}
0 & \text { normal trade } \\
-1 & \text { malicious behaviour }
\end{array}\right.
$$

If $\mathrm{D}_{\mathrm{ij}}$ and $\mathrm{N}_{\mathrm{ij}}$ all above the system set the boundary value, then the node $\mathrm{j}$ can be identified as a credible node. It can make transaction without $\left(\mathrm{R}_{\mathrm{j}}\right)$.

\section{Recommendation Trust Value}

We need to consider $R_{j}$ when the peers don't trade directly or $N_{i j}$ is too low or $D_{i j}$ don't reach the threshold value of system. In order to response the recommendation trust value accurately, we introduce the accuracy factor $(\mathrm{A}(\mathrm{x}))$.

Definition 2. The calculation formula of $R_{j}$ is:

$$
\mathrm{R}_{\mathrm{j}}=\sum_{\mathrm{x}} \mathrm{R}_{\mathrm{x} \rightarrow \mathrm{j}} \times \mathrm{A}(\mathrm{x})
$$

Where $\sum_{x} R_{x \rightarrow j}$ means a congregation that all the peers of recommendation information with node $\mathrm{j}$ transaction records.

Definition 3. Calculation formula of offset factor $d_{x}$ is:

$$
\mathrm{d}_{\mathrm{x}}=\left[\sum_{\mathrm{u} \in \operatorname{IS}(\mathrm{i}) \cap \mathrm{IS}(\mathrm{x})}\left|\mathrm{S}_{\mathrm{iu}} / \mathrm{N}_{\mathrm{iu}}-\mathrm{S}_{\mathrm{xu}} / \mathrm{N}_{\mathrm{xu}}\right|\right] /\left[\mathrm{IS}_{\mathrm{iu}} \cap \mathrm{IS}_{\mathrm{xu}}\right]
$$

Where IS $S_{i u}$ represents the congregation ofi $\leftrightarrow \mathrm{u}, I S_{i u} \cap I_{\text {xu }}$ represents all the common peers of $\mathrm{i} \leftrightarrow \mathrm{x}$. Obviously, $0 \leq \mathrm{d}_{\mathrm{x}} \leq 1$. IfIS $\mathrm{IS}_{\mathrm{iu}} \cap \mathrm{IS}_{\mathrm{xu}}=0$, that means there is no common node ofi $\rightarrow \mathrm{x}$, let the initial valued $_{\mathrm{x}}=0.5$.

Definition 4. The calculation formula for accuracy factor of feedback node is:

WhereA $(x) \leq 1 ; s=1,2,3 \cdots$

$$
\mathrm{A}(\mathrm{x})=1-\mathrm{d}_{\mathrm{x}}{ }^{1 / \mathrm{s}}
$$

$\mathrm{A}(\mathrm{x})$ is low enough, then the credibility of recommendation peer should be suspected. So, we set the boundary value, ifA $(x) \leq 0.1, R_{j}$ can be neglected.

\section{The risk Value}

It exist a certain risk when any entity (node) interact with others in a virtual environment. So the calculation formula of risk value is: 


$$
\mathrm{R}(\mathrm{x})=\xi \mathrm{D}_{\mathrm{ij}}+(1-\xi) \sum_{\mathrm{x}} \mathrm{R}_{\mathrm{x} \rightarrow \mathrm{j}} \times \mathrm{A}(\mathrm{x})
$$

Where $\xi$ is a weight, it should be set a relatively small value. Because of dynamic and heterogeneous of peers in $\mathrm{P} 2 \mathrm{P}$ network, it is difficult to find the credible third party. The risk value of $d_{x}$ is larger than $D_{i j}$.

\section{The global reputation value}

Definition 5. The calculation formula of global reputation value of node $\mathrm{j}$ is:

$$
\mathrm{T}_{\mathrm{i} \rightarrow \mathrm{j}}=\mu \mathrm{D}_{\mathrm{ij}}+(1-\mu) \mathrm{R}_{\mathrm{j}}-\mathrm{R}(\mathrm{x})
$$

Where $\mu$ represents a weighted factor according to its trading experience.

We discuss the following cases:

1) In case, $\mu=0$. The node itself has no definite view. Its trust value mainly depends on other peers' recommendation feedback.

2) In case, $\mu=1$. The node only believes the history experience of itself. It is skeptical the feedback information of other recommendation peers.

3) In case, $0<\mu<1$.at beginning $\mathrm{T}_{\mathrm{i} \rightarrow \mathrm{j}}$ is limited, so $\mu$ is a little proportion, with the accumulation of trading, the peers are more likely to trust their own experience. Therefore, the weighted factor is dynamic change. Let

$$
\mu=1-\sigma^{\mathrm{k} / \mathrm{n}}
$$

Where the value ranges of $\sigma$ is $0.5<\sigma<1$; $\mathrm{k}$ shows the $\mathrm{k}$ time transactions; $\mathrm{n}$ shows total number of transactions and value range is $\mathrm{n} \in$ $\{1,2,3 \cdots\}$. Thus in this case, $\mu$ we get is fit for the situation and mode of actual transaction. Therefore, formula of $T_{i \rightarrow j}$ is:

$$
\mathrm{T}_{\mathrm{i} \rightarrow \mathrm{j}}=\mu \mathrm{D}_{\mathrm{ij}}+(1-\mu) \sum_{\mathrm{x}} \mathrm{R}_{\mathrm{x} \rightarrow \mathrm{j}} \times \mathrm{A}(\mathrm{x})-\mathrm{R}(\mathrm{x})
$$

Finally, we compare $T_{i \rightarrow j}$ with threshold value of system $\left(T_{i \rightarrow j 0}\right)$. If $T_{i \rightarrow j}>T_{i \rightarrow j 0}$, then the node is credible and can be traded; otherwise, the node isn't credible.

\section{The Experiment and Analysis}

In this section, we will assess the performance of our recommendation trust model as compared to the Eigen Trust model and Peer Trust model in P2P network. Take the downloading files for instance.

\subsection{The Simulation Experiment}

Experimental environment: the number of peers is 100; total number of files is 500 . We distribute 500 documents to 100 peers randomly, and make sure that each node has a document at least. The node has this file if it downloads successfully; otherwise, the peer downloads failing. We set that the number of honest peers is more than the number of malicious peers, and the first transaction request is launched by honest nodes. 


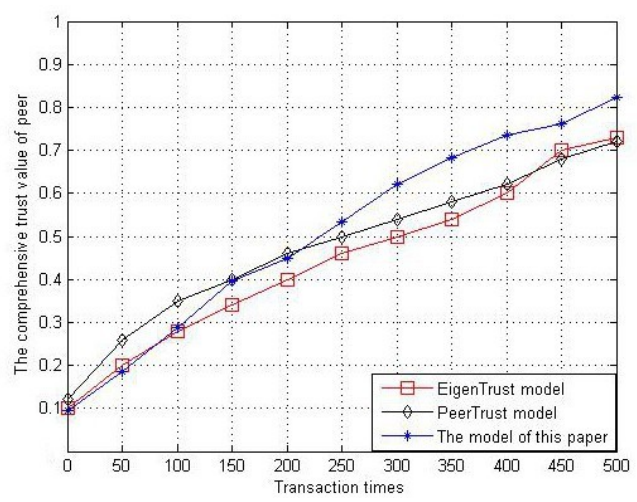

Fig. 1. The influence of transaction times to trust value

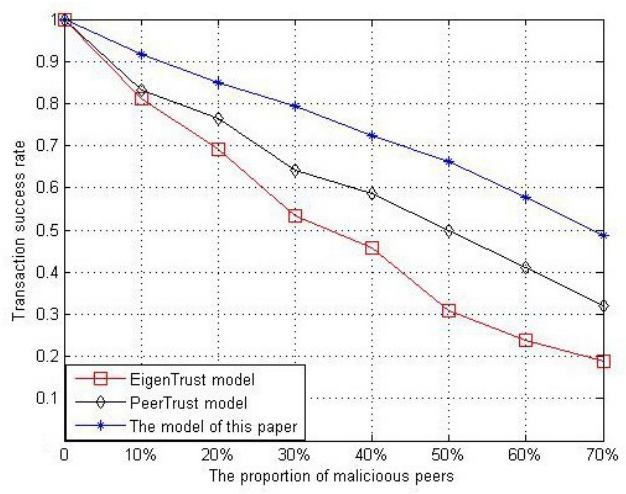

Fig. 2. Transaction success rate under different proportion of malicious peers

\subsection{The Analysis}

Figure 1: Eigen Trust model not consider the accuracy of recommendation peers, with the accumulation of transactions, $\mathrm{T}_{\mathrm{i} \rightarrow \mathrm{j}}$ is increasing. PTM evaluates the reliability of peers by depending on other peer's recommendation completely, but it ignores the personal interactive historical experience. The model of this article considers transaction record and accuracy of recommendation peers. Therefore, $\mathrm{T}_{\mathrm{i} \rightarrow \mathrm{j}}$ of target peer is real. Experimental results show that the accuracy and validity of the model.

Figure 2: After 50 transactions on average, we set the honest peer to evaluate other peers. From the results, the transaction success rate of each model has different degree dropping with gradually increasing in the proportion of the malicious peers. When the number of malicious peers is more than $40 \%$, the error rate is high in Eigen Trust model and transaction success rate falls rapidly. For Eigen Trust model, it is almost hard to run if there are many malicious peers in environment. But no matter the size of proportion of malicious peers, PTM and the model of this article still remain relatively high transaction success rate. The results confirmed that the model has a certain safety and can play a role in the real application. 


\section{Conclusion}

We have presented a trust model to improve for existing problems of Eigen Trust model in P2P network. The model considers the credibility of recommendation peers and prevents the collaborative cheating of malicious peers effectively. In P2P simulation, the results show that the model is effective and practical. But we not involved in some problems such as the cost of system about calculation trust value of peer, timeliness, etc. We hope that in the work further study.

Acknowledgement. This work is supported by Open Foundation of Guangxi Key Laboratory of Trusted Software (Guilin University of Electronic Technology).

\section{References}

1. Yu, D.-G., Chen, N., Tan, C.-X.: Research on Trust Cloud-based Subjective Trust Management Model under Open Network Environment. Information Technology 10(4), 759$768(2011)$

2. Li, X., Zhou, F., Yang, X.: A multi-dimensional trust evaluation model for large-scale P2P computing. Journal of Parallel and Distributed Computing 71(6), 837-847 (2011)

3. Teacy, W.T., Jigar, P., Jennings, N.R.: TRAVOS: Trust and Reputation in the Context of Inaccurate Information Sources. Autonomous Agents and Multi-agent Systems 12(2), 183-198 (2006)

4. Kamvarsd, Schlosser, M.T.: Eigen Rep: reputation management in P2P networks. In: Proc of the 12th International World Wide Web Conference Budapest, pp. 123-134. ACM Press, New York (2003)

5. Guo, C., Li, M.-C., Yao, H.-Y.: The trust model based on recommended under P2P network. Computer Engineering 34(24), 157-159 (2008)

6. Xiong, L., Liu, L.: Peer Trust: supporting reputation-based trust for peer-to-peer electronic communities. IEEE Trans. on Knowledge and Data Engineering 16(7), 843-857 (2004)

7. Dou, W., Wang, H.-M., Jia, Y., Zou, P.: A recommendation-Based Peer-to-Peer Trust Model. Journal of Software 15(4), 571-583 (2004) 